\section{Fractured laryngeal mask airway (LMA)}

To the Editor:

In a 65-yr-old man undergoing elective cystolithopaxy anesthesia was induced and a size 4 LMA (Intavent, Oxon, UK) inserted. At emergence, the patient bit on the LMA shaft, shearing it into two pieces (Figure). The proximal shaft outside his clenched teeth was severed. The distal shaft and the inflated LMA remaining inside the patient's mouth. A facemask with oxygen was applied. He maintained spontaneous breathing but did not open his mouth. One minute later, his mouth opened and the LMA was removed using McGill forceps after cuff deflation.

Examination of the LMA showed a spiral fracture of the distal shaft and marked yellowish discoloration of the shaft. The manufacturing date was the second quarter of 1991. Autoclaving (steam, $134^{\circ} \mathrm{C}$ ) at our hospital conforms to the manufacturer's recommendations. ${ }^{1}$ We estimate that this LMA had been autoclaved over 400 times. The Canadian supplier (Vitaid, Toronto) recommended the following to ensure safety: ${ }^{1,2}$ first, record each autoclaving and discard after 40 uses; second, before each use, check colour of shaft, aperture bars, competency of pilot balloon valve, competency of LMA cuff, $15 \mathrm{~mm}$ connector fit; third, flex the shaft $180^{\circ}$ and observe for kinking; fourth, use a bite block.

This case illustrates the potential risk of a fractured LMA due to excessive re-use. Although dramatic in presentation, the patient had no sequlae. It is important to retrieve all pieces of the fractured LMA and that none is aspirated into the lungs.

David T. Wong MD

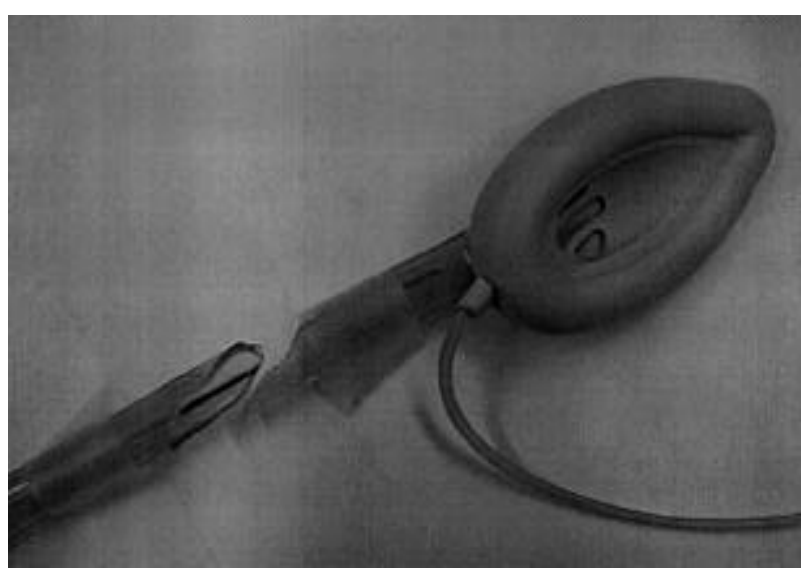

FIGURE
Glenn P. McGuire MD

Toronto, Canada

References

1 Brimacombe JR, Brain AIJ, Berry AM. Preparation for use. In: The Laryngeal Mask Airway. A Review and Practical Guide. London: W.B. Saunders Company, 1997: 52-65.

2 Brain AIJ. The Laryngeal Mask Airway, Instruction manual. Henley, UK: Intavent Research Ltd, 1995: 41-2.

REPLY:

Thank you for the opportunity to respond to Dr. David Wong's letter concerning overuse of the laryngeal mask airway (LMA).

The LMA is a reusable, but consumable device. Performance tests were carried out by the manufacturer (Laryngeal Mask Company Ltd., Cyprus, available from Vitaid Ltd., Toronto). With each use, the silicone material degrades and loses strength. Under typical conditions, the mechanical properties of the LMA dropped off significantly after 40 uses. To this end, a 40 use record card is supplied with each LMA. The card allows for recording of the serial number and cleaner's initials. The LMA is warranted from defects in manufacture to 40 uses or one year from the date of purchase.

The laryngeal mask is a robust device, but no end point in its life cycle has been previously discussed. We feel that clinicians should monitor and inspect the LMA before each use to ensure patient comfort and safety. Details of the inspection procedures are contained in the instruction manual.

Finally, the use of a bite block and properly taping the airway tube are essential to having a secure airway with the LMA.

William G. Stewart

President, Vitaid Ltd.

Toronto, Ontario

\section{Subcutaneous tunneling of the intersca- lene catheter.}

To the Editor:

Patient-controlled interscalene analgesia (PCIA) by means of an interscalene catheter offers excellent pain relief after major shoulder surgery ${ }^{1}$ but, with time catheter dislocation occurs. Boezaart et al. ${ }^{2}$ described a technique to fix the interscalene catheter by making a subcutaneous tunnel with the aid of a 17 G Tuohy needle. We also perform subcutaneous tunneling for the 
interscalene catheter. After skin infiltration with 3-4 ml lidocaine $1 \%$, an $18 \mathrm{G} i v$ cannula is inserted subcutaneously just above the suprasternal notch and then advanced cranially at an angle of 45 to exit 3-4 mm above the primary catheter insertion point. The proximal end of the catheter is threaded retrogradely through the $i v$ cannula, before the latter is removed, so that the catheter finally is tunneled $4-5 \mathrm{~cm}$ subcutaneously. The catheter is fixed with usual dressing tapes.

Using this method described in 100 patients, the catheter was used for three days in $80 \%$ of the patients, for two days in $10 \%$ and in the remaining $10 \%$ for four days. The subcutaneous catheter tunnel was well accepted by all patients. Our findings support Boezaart et al. ${ }^{2}$ We agree with their method of securing the catheter subcutaneously, but we recommend not to use a large and inflexible $17 \mathrm{G}$ Tuohy needle for this purpose, but rather a thinner and more flexible 18 G $i v$ cannula, which may reduce potential bleeding or damage to the small sensory cutaneous nerves while providing a very low incidence of catheter dislocation.

Georgios Ekatodramis MD

Alain Borgeat MD

Zurich, Switzerland

\section{References}

1 Borgeat A, Schäppi B, Biasca N, Gerber C. Patient-controlled analgesia after major shoulder surgery: patientcontrolled interscalene analgesia versus patient-controlled analgesia. Anesthesiology 1997; 87: 1343-7.

2 Boezaart AP, de Beer JF, du Toit C, van Rooyen K A new technique of continuous interscalene nerve block. Can J Anesth 1999; 46: 275-81. 